\title{
Artificial Intelligence: Help or Hindrance for Family Physicians?
}

\author{
Farhana Irfan
}

\begin{abstract}
The use of Artificial Intelligence (Al) and related technologies is rapidly increasing and its application in clinical practice is a promising area of development. Artificial Intelligence can be a solution in the future as a physician's new assistant; Al-physician combinations can act like models of 'peaceful co-existence'. While it has the potential to mold many dimensions of patient care and can augment quality improvement, it cannot replace a family physician's diagnostic intelligence, empathy and relationships. Physicians need to strike a balance between these combinations for better health outcomes without increasing patients' frustration.
\end{abstract}

KEYWORDS: Artificial Intelligence, Family Physicians, Machine learning.

How to cite this:

doi: https://doi.org/10.12669/pjms.37.1.3351

Irfan F. Artificial Intelligence: Help or Hindrance for Family Physicians? Pak J Med Sci. 2021;37(1):288-291. doi: https://doi.org/10.12669/pjms.37.1.3351

This is an Open Access article distributed under the terms of the Creative Commons Attribution License (http://creativecommons.org/licenses/by/3.0), which permits unrestricted use, distribution, and reproduction in any medium, provided the original work is properly cited.

The use of Artificial Intelligence (AI) and related technologies is rapidly increasing. What is Artificial Intelligence? It is not one technology, but rather a collection of techniques that allow computers to work and react like humans by problem-solving. Its use is prevalent in almost all fields and is beginning to be applied in healthcare too. ${ }^{1} \mathrm{AI}$ has the potential to mold many dimensions

1. Farhana Irfan

King Saud University Chair for Medical Education Research

and Development, Department of Family

and Community Medicine,

College of Medicine,

King Saud University,

Riyadh, Saudi Arabia.

Correspondence:

Farhana Irfan,

King Saud University Chair for Medical Education

Research and Development,

College of Medicine,

King Saud University,

Department of Family and Community Medicine (34),

PO Box 2925, Riyadh 11461,

Kingdom of Saudi Arabia.

E-mail: masoodfarhana1969@gmail.com

* Received for Publication:

July 23, 2020

* Revision Received:

* Revision Accepted: of patient care but we have yet to grasp fully the speed and breadth of this new revolution. At present, $\mathrm{AI}$ is being utilized in the medical field by being incorporated into health systems; varying from simple scheduling of appointments, online medical records, reminders for follow-ups to diagnosis in the fields of radiology, pathology, and ophthalmology. ${ }^{2,3}$ The field of Family Medicine is also evolving rapidly to meet growing patient demands such as catering to patients of all age groups with chronic or acute illnesses, or both. In general, the family physicians are the first to see patients, when the illness is still in the process of development. They are expected to diagnose individuals earlier on and avoid excessive testing and referrals., ${ }^{4,5}$ Currently, a major amount of a doctor's time is spent on computer work or on other administrative tasks, instead of with patients. ${ }^{6}$ The "art" of Family medicine is being lost. Physicians feel increasingly stuck with desk work. Patients are not "looked at" anymore, giving a feeling that doctors are obsessively fixated on information, charts and monitors. This has led to erosion in the doctor-patient relationship over recent years. With increasing life expectancies, the patient load will further increase and so will the 
computer work in the coming years. The question arises; how we can give more time to patients without compromising the quality and patient care? Artificial Intelligence can be a solution in the future as a physician's new assistant.

In healthcare, AI studies suggest productivity gains $^{7,8}$ and there is a growing interest in using AI to augment and improve the practice globally. However, there is limited literature available on its use in low resource settings where its application is nascent and its voice needs to be amplified to shape its future. Developing countries grapple with high burden of disease as well as with the poor healthcare infrastructure. A local survey on the use of AI in healthcare in low resource settings reported lack of trained AI professionals as the most common (64\%) hurdle followed by difficulty in identifying use cases $(28 \%)$. It's no surprise that the medical community might not be familiar with its current applications possible in the country. A PubMed search revealed AI publications in the field of medical education ${ }^{9}$ and across various specialties of medicine ${ }^{10-13}$ but none in the discipline of Family Medicine locally.

The degree to which AI can improve primary care is undetermined, a review of its application at the primary care level will accelerate realizing of the full potential for improving the healthcare thus making it achievable.

There are significant hypothesis on the capacity of AI, which are still developing. The main purpose can be improving processes and relieving some of the physician's workload. Additionally, the possible areas that can be covered are; improved quality of care on routinely collected administrative data (such as triaging patient inquiries and processing patient claims) and assistance for physicians by providing up-to-date medical information from journals, textbooks and clinical practices, so that they can be informed on proper patient care. ${ }^{14,15}$

Clinical documentation is becoming mandatory worldwide and consumes a lot of time on a daily basis. Taking patient histories and making decisions with a standard computer is time consuming and distracts physicians from paying attention to the patient. The data that is obtained towards the end of consultation is either dismissed or ignored. As a consequence, GPs may unfortunately produce a misrepresented document with a distorted range of differential diagnoses. ${ }^{16}$ Unlike physicians, AI systems never get tired or irritable. Machine learning can be used for natural language processing, speech recognition and text analysis. It can transcribe patient interactions, analyze unstructured clinical notes on patients and give a probable diagnosis. It could assist GPs in recognizing important aspects and overcoming cognitive biases, thus guiding about tests and reasonable treatment options. ${ }^{17-20}$ Clinicians can discuss more with the patients if AI takes over the mundane data-entry tasks. ${ }^{21}$

In addition, symptom checkers can serve in helping educate patients on the range of diagnoses that might fit their symptoms. The triage function informs patients whether they should seek care at all and, if so, where and with what urgency, empowering the patients with self-treatable conditions rather than feeling dependent on their physicians. ${ }^{22}$ Audit of vignette based symptom checkers has shown to provide appropriate advice for up to $80 \%$ for emergency cases. ${ }^{23}$ It can also screen patients, supplement the triage lines, manage a preliminary analysis suggesting likely diagnosis (thus reducing the number of visits, which can save patients' time and money) and in addition, may decrease demand on primary care providers. . $^{1,414,16-18,20}$

AI can have a considerable role in preventative medicine by summarizing a large set of medical information and using algorithms to proactively propose consultations, suggesting optimal treatments. It can also bring specialist skills into primary care by early diagnosis of diseases and recognition of high risk patients by pattern recognition in imaging results such as automated diagnosis of diabetic retinopathy, interpreting radiographs and ultrasound. ${ }^{24-26}$ Relevant patient education material and reminders can be effective in personalizing care and can be very promising in the future. It can also be a good option to support large under resourced populations where human expertise is scarce. . $^{26-28}$

It is often seen that communication skills are a major reason for patient dissatisfaction. There is concern that these skills remain basic even after training. There is evidence that communications skills can be effectively developed but lack of feedback on performance is an obstacle for improvement; primarily as it is time consuming for physicians. AI has a prospective role for assessing and training physician's communication skills by 
analyzing the recorded consultation and providing feedback on the delivery such as proportion of talk by patient and clinician, overlapping talk, speed of speech, tone of voice and language, clinical jargons etc. for improvement. ${ }^{17}$

Furthermore, what does AI mean to general practitioners and for primary care? It does have the potential to bring significant change to healthcare but, there is contemplation on the risks too. Like any other innovation, it requires careful thought. AI has a significant amount of hype associated with it, which needs to be evaluated before complete adoption. This matter requires discussion. Can AI replace clinical experience and human decision? Can AI have the sixth sense that physicians feel in challenging cases? Can the machines understand the family medicine patient care context? Can it replace a doctor's clinical diligence? There will always be the lingering threat of over or under diagnoses. All this requires having humans (physicians) at the center.

These applications are designed to amplify human cognition and work, rather than replace it; they can be better calculators but not better thinkers. AI-physician combinations should act like models of 'peaceful co-existence', like autopilots on planes; improved safety without compromising the training of pilots. ${ }^{29}$ It can free physicians from spadework and let them focus on the humanistic elements of care and practice in line with the roots of medicine as a healing profession. Another obstacle is that it has to be tuned to the correct data, from the appropriate population. AI diagnostic tools developed in one country are not applicable worldwide and will not provide the correct guidance to a primary care setting elsewhere, as one size does not fit all.

At this moment, the physicians lack the expertise required to use AI in family practice. This raises an important question about the sufficiency of medical curricula to equip future physicians for the advancement in clinical practice. Medical schools need to incorporate courses on AI in medical school curriculum to increase the awareness as well as highlight the importance of AI during undergraduate as well as postgraduate training in medical education ${ }^{9}$ as improvements in education, may improve the rift. Family medicine training programs need to deploy appropriate training and educational programs for physicians on how to use data science and AI for better services. Family practice can embrace it and evaluate it as any new medical innovation and avail the scope it offers. Physicians need to strike a balance between these combinations for better health outcomes without increasing the frustration of patients.

This is a preliminary descriptive analysis which provides insights into the ways technological advancements can impact the primary care, the perceived limitations and benefits. This must be read with a futuristic and contemporary vision as a bridge to fill in the gaps in the fragmented knowledge that have been elucidated. If it is rightly and timely adopted it can help physicians to achieve better patient care.

To conclude, AI can augment quality improvements in health care at reduced costs, but it cannot replace a family physician's diagnostic intelligence, empathy and relationships ${ }^{2}$. The question arises; can AI incorporate and respond to visual clues and personal preferences, maintain patient dignity and cultural and social demands, while also overcoming undocumented, immeasurable events and ethical concerns? Physicians will remain important and should become tech savvy to work side by side with it; as the patient population will continue to grow in the foreseeable future.

Ethics approval: Not applicable

Consent for publication: Not applicable

Competing Interests: The author has no competing interests.

Acknowledgments: This work was supported by the Deanship of Scientific Research, King Saud University through Vice Deanship of Scientific Research Chair. I warmly thank my daughter Syeda. Fatima who read the manuscript and made some stylistic suggestions.

\section{REFERENCES}

1. Ross J, Webb C, Rahman F, Artificial Intelligence in Healthcare. Academy of Medical Royal Colleges, 2019. Available from: https://www.aomrc.org.uk/wpcontent/uploads/2019/01/Artificial_intelligence_in_ healthcare_0119.pdf. Accessed on 16 March 2020.

2. Magrabi F, Ammenwerth E, McNair JB, De Keizer $\mathrm{NF}$, Hypponen $\mathrm{H}$, Nykanen $\mathrm{P}$, et al. Artificial Intelligence in Clinical Decision Support: Challenges for Evaluating AI and Practical Implications. Yearb Med Inform. 2019;28(1):128-134. doi: 10.1055/s-0039-1677903.

3. Amisha Malik P, Pathania M, Rathaur VK. Overview of artificial intelligence in medicine. J Family Med Prim Care. 2019;8(7):2328-2331. doi: 10.4103/ jfmpc. jfmpc_440_19

4. Summerton N, Cansdale M. Artificial intelligence and diagnosis in general practice Br J Gen Pract 2019;69(684):324325. doi: 10.3399/bjgp19X704165 
5. Upshur R. Artificial Intelligence, Machine Learning and the potential impacts on the practice of Family Medicine: A briefing document. Available from: https://www. cfpc.ca/uploadedFiles/About_Us/Discussion-Paper-AIImpacts-on-FM-AMS-CFPC-May2019.pdf). Accessed on 19 March 2020

6. Sinsky C, Colligan L, Li L, Prgomet M, Reynolds S, Goeders L, Westbrook J, Tutty M, Blike G.Allocation of Physician Time in Ambulatory Practice: A Time and Motion Study in 4 Specialties. Ann Intern Med. 2016;165(11):753-760. doi: 10.7326/M16-0961

7. Cronin S, Doherty G. Touchless computer interfaces in hospitals: A review. Health Informatics J. 2019;25(4):13251342. doi: $10.1177 / 1460458217748342$

8. Hodgson T, Magrabi F, Coiera E. Efficiency and safety of speech recognition for documentation in the electronic health record. J Am Med Inform Assoc. 2017;24(6):11271133. doi: $10.1093 /$ jamia/ocx073

9. Imran N, Jawaid M. Pak J Med Sci. 2020;36(5):857-859. doi: 10.12669 / pjms.36.5.3042

10. Hoodbhoy Z, Hasan B, Siddiqui K. Does artificial intelligence have any role in healthcare in low resource settings? J Med Artif Intell. 2019;2:13.

11. Abid S, Awan B, Ismail T, Sarwar N, Sarwar G, Tariq M, et al. Artificial intelligence: medical student's attitude in district Peshawar Pakistan. Pak J Public Health. 2019;9(1):19-21. doi: 10.32413/pjph.v9i1.295

12. Shaikh Q, Kamal AK. Can telemedicine improve stroke outcomes? J Pak Med Assoc. 2012;62(12):1352-1353.

13. Abbasi AN, Khan AMH, Qureshi BM. Radiomics - Using Artificial Intelligence in the Quest towards Personalised Radiation Treatment. J Coll Physicians Surg Pak. 2019;29(2):99-100. doi: 10.29271/jcpsp.2019.02.99

14. Semigran HL, Linder JA, Gidengil C, Mehrotra A4. Evaluation of symptom checkers for self diagnosis and triage: audit study. BMJ. 2015;351:h3480. doi: 10.1136/ bmj.h3480

15. Jiang F, Jiang $\mathrm{Y}$, Zhi $\mathrm{H}$, et al. Artificial intelligence in healthcare: past, present and future. Stroke Vasc Neurol. 2017;2(4):230-243. doi: 10.1136/svn-2017-000101

16. Campbell JL, Fletcher E, Britten N, Green C, Holt TA, Lattimer V. Telephone triage for management of same-day consultation requests in general practice (the ESTEEM trial): a cluster randomised controlled trial and cost-sequence analysis. Lancet. 2014;384(9957):1859-1868. doi: 10.1016/S0140-6736(14)61058-8

17. Ryan P, Luz S, Albert P, Vogel C, Normand C, Elwyn G. Using artificial intelligence to assess clinicians' communication skills. BMJ. 2019;364:1161. doi: 10.1136/ bmj.1161

18. Barbieri C, Molina M, Ponce P, Tothova M, Cattinelli I, Ion Titapiccolo J. et al. An international observational study suggests that artificial intelligence for clinical decision support optimizes anemia management in hemodialysis patients. Kidney Int. 2016;90(2):422-429. doi: 10.1016/j. kint.2016.03.036
19. Krittanawong C, Zhang H, Wang Z, Aydar M, Kitai $\mathrm{T}$. Artificial intelligence in precision cardiovascular medicine. J Am Coll Cardiol. 2017;69(21):2657-2664. doi: 10.1016/j.jacc.2017.03.571

20. Davenport T, Kalakota R. The potential for artificial intelligence in healthcare. Future Healthc J. 2019;6(2):9498. doi: 10.7861/futurehosp.6-2-94

21. Matheny MS, Israni T, M Ahmed, D Whicher, Editors. 2019. Artificial Intelligence in Health Care: The Hope, the Hype, the Promise, the Peril. NAM Special Publication. Washington, DC: National Academy of Medicine.

22. Semigran HL, Linder JA, Gidengil C, Mehrotra A. Evaluation of symptom checkers for self- diagnosis and triage: audit study. BMJ. 2015;351:h3480.

23. Evaluation of symptom checkers for self-diagnosis and triage: audit study. BMJ. 2015;351:h3480. doi: 10.1136/ bmj.h3480

24. Abramoff MD, Lavin PT, Birch M, Shah N, Folk JC. Pivotal trial of an autonomous AI-based diagnostic system for detection of diabetic retinopathy in primary care offices. NPJ Digit Med. 2018;1(01):39.

25. Gulshan V, Peng L, Coram M, Stumpe MC, Wu D, Narayanaswamy A, et al. Development and validation of a deep learning algorithm for detection of diabetic retinopathy in retinal fundus photographs. JAMA. 2016;316(22):24022410. doi: 10.1001/jama.2016.17216

26. Buch VH, Ahmed I, Maruthappu M. Artificial intelligence in medicine: current trends and future possibilities. $\mathrm{Br}$ J Gen Pract. 2018;68(668):143-144. doi: 10.3399/ bjgp18X695213

27. Liyanage $\mathrm{H}$, Liaw ST, Jonnagaddala J, Schreiber R, Kuziemsky C, Terry AL, de Lusignan S. Artificial Intelligence in Primary Health Care: Perceptions, Issues, and Challenges. Yearb Med Inform. 2019;28(1):4146. doi: 10.1055/s-0039-1677901

28. Liyanage $\mathrm{H}$ et al. Artificial Intelligence in Primary Health Care: Perceptions, Issues, and Challenges. Primary Health Care Informatics Working Group Contribution to the Yearbook of Medical Informatics 2019. Available from: https://www.researchg ate.net/publication/332682710_ Artificial_Intelligence_in_Primary_Health_Care_Perceptions_Issues_and_Challenges_Primary_Health_ Care_Informatics_Working_Group_Contribution_to_th e_Yearbook_of_Medical_I nformatics_2019. DOI: 10.1055/ s-0039-1677901 [accessed Mar 19, 2020].

29. Ross J, Webb C, Rahman F. Artificial Intelligence in Healthcare. Academy of Royal Medical Colleges 2019. Available from: https://www.a omrc.org.uk/wp-content/uplo ads/2019/01/Artifici al_intelligence_in_health care_0119. pdf [acc essed Mar 19, 2020]. 